\title{
Presença e Ausência do ut Completivo em Plauto: A Estrutura, Alguma Bibliografia e Dados GLOBAIS
}

\author{
Alex Mazzanti Jr. ${ }^{1}$
}

\begin{abstract}
RESUMO: Este artigo tem como objeto o estudo da presença e ausência do ut completivo em Plauto, com os verbos principais facio e uolo complementados por subjuntivo. Primeiramente, discuto a estrutura em questão, fazendo a diferenciação entre duas possíveis leituras dos termos "parataxe" e "hipotaxe". Por conta da polêmica indefinição do termo "parataxe", opto por sempre utilizar “justaposição" para me referir à estrutura em questão quando sem uso da conjunção. Em seguida, apresento a análise de dois autores que apresentam recolhas que visam ser exaustivas dos exemplares, mas que analisaram a questão sob outra perspectiva e com outros objetivos. Por fim, apresento os resultados globais quantitativos de minhas próprias recolhas relativas à complementação de subjuntivo dos verbos facio e uolo em Plauto. Embora ambos os verbos apresentem exemplares ora com e ora sem o $u t$, a distribuição da estrutura tem números opostos, evidenciando a necessidade de que sejam analisados separadamente.
\end{abstract}

PALAVRAS-CHAVE: Plauto; Latim arcaico; Parataxe; Justaposição; Sintaxe.

ABSTRACT: This paper aims to study the presence and absence of the conjunction $u t$ in substantive clauses in Plautus. The main verbs facio and uolo, when followed by subjunctive, are considered. Firstly, there is a discussion about the structure researched. A difference between the two possible interpretations of parataxis and hypotaxis is made. Owing to the controversy of the term "parataxis", I chose to always use "juxtaposition" to refer to this structure in the absence of the conjunction. Then I analyse the texts of two scholars who have done similar complete collections of tokens, but that have made their analysis with other perspectives and objectives. Finally, I present the general quantitative figures of my own collection of tokens of subjunctive complementation of facio and uolo in Plautus. Although both verbs have tokens with and without $u t$, the distribution of it has opposite figures, making clear the necessity of separate analysis.

KEYWORDS: Plautus; Archaic Latin; Parataxis; Juxtaposition; Syntax.

\footnotetext{
${ }^{1}$ Mestrando pelo Programa de Pós-graduação em Letras Clássicas, FFLCH/USP. Essa pesquisa teve o apoio da Fundação de Amparo à Pesquisa do Estado de São Paulo (FAPESP), processo ${ }^{0}$ 2015/26060-5. E-mail: alexmazzantijr@gmail.com.
} 
No chamado latim clássico, baseado especialmente nos textos de Cícero e César, no século I a.C. (e um pouco além), encontramos um sistema linguístico, seja no âmbito morfológico quanto sintático, mais simétrico e padronizado. Essa normalização se opõe à variação encontrada em períodos anteriores, dos quais me interessa o que chamarei de latim arcaico, geralmente identificado com os séculos III e II a.C. Vejamos a sugestão de periodização empregada por Weiss (2009, p. 23), das quais seleciono as que nos interessam:

B. Old Latin (OL): 3rd-2nd century. We have a considerable number of texts from this period, both epigraphical and literary (most importantly Plautus, Cato, and the fragments of Livius Andronicus, Naevius, Ennius, Pacuvius, and Lucilius).

C. Classical Latin (CL): $1^{\text {st }}$ century BCE to $3^{\text {rd }} / 4^{\text {th }}$ century CE. In the works of Cicero and Caesar the morphological and syntactic variation found in earlier Latin has been greatly reduced. The norms developed in this period became fixed standard for many centuries. ${ }^{2}$

Desse modo, enquanto no latim clássico o comportamento em geral das estruturas sintáticas é muito mais previsível por conta dessa padronização, uma série de estruturas do latim arcaico, embora já notadas e até classificadas em estudos sobre a língua arcaica, não foram detalhadamente descritas e explicadas, como no caso do uso da conjunção ut num contexto com verbo no subjuntivo: enquanto no latim clássico há uma série de estruturas sintáticas que usam subjuntivo que são obrigatoriamente introduzidas pela conjunção ut, tendo como exceção a isso alguns usos estilísticos, no latim arcaico a conjunção ora está presente, ora está ausente, ocasionando um uso que na literatura é chamado de paratático e que preferirei chamar de justaposto.

A seguir, discutirei a estrutura como um todo, tendo já como enfoque o recorte que farei mais à frente, das orações completivas que podem apresentar ou não a conjunção ut, tendo como verbos principais facio e uolo em Plauto, principal corpus do latim arcaico. Após, exemplifico o tratamento que a bibliografia fez

\footnotetext{
${ }^{2}$ Em tradução: "B. Latim Arcaico: séculos III-II [a.C.]. Nós temos um número considerável de textos desse período, ambos epigráficos e literários (os mais importantes Plauto, Catão e fragmentos de Lívio Andronico, Névio, Énio, Pacúvio e Lucílio). C. Latim Clássico: século I a.C. ao século III/IV d.C. Nas obras de Cícero e César, a variação morfológica e sintática encontrada no latim mais antigo foi muito reduzida. As normas desenvolvidas nesse período se tornaram um padrão fixo por muitos séculos.”
} 
sobre o assunto, notadamente em Durham (1901) e Bennett (1982), e, então, apresento os dados globais consolidados para os dois verbos, que permitem ver o comportamento global das estruturas em Plauto.

Teorizando parataxe e justaposição: compreendendo a estrutura

Ao longo da tradição de estudos do latim, o termo "parataxe" tem sido usado com uma enorme variação de definições, muitas vezes não muito esclarecidas pelos autores, incluindo, por exemplo, (i) a mera coordenação assindética: duas orações de mesmo estatuto sintático, ou seja, nenhuma exerce função sintática na outra, e sem o uso de conjunção alguma, como em Plaut. Most. 566 hic ad me it, saluos sum, "ele vem até mim, estou salvo"; ou ainda (ii) pode ser utilizado para designar orações que, embora possam ser entendidas como subordinadas por utilizarem geralmente o subjuntivo e exercerem alguma função sintática relacionada a um verbo dito principal, não estão conectadas por uma conjunção ou outra partícula subordinante, estando, portanto, justapostas à oração principal (como em Plaut. Cas. 544 uin uocem? "você quer que eu [a] chame?"). Sendo aplicado pela tradição a uma gama tão diversa de estruturas, o termo "paratático" foi e ainda pode ser objeto de polêmica, de modo que utilizo sempre a terminologia derivada do termo "justaposição": enquanto o termo "paratático" gera ambiguidade quanto ao julgamento do estatuto sintático (Deve ser aplicado somente onde não há subordinação? Pode também ser aplicado quando há subordinação não evidenciada pelo subordinador?), o termo "justaposto" simplesmente descreve a ausência de um mediador, sem entrar no mérito do estatuto sintático dos termos, o que é funcional para esta pesquisa, já que ela visa justamente verificar a presença ou ausência do ut completivo.

De qualquer modo, para deixar claro qual é meu objeto de estudo, coloco mais alguns exemplos do que acabamos de discutir:

(1) Parataxe "real" ou sentenças assindéticas:

(a) Tantas diuitias habet; nescit quid faciat auro

(Plaut. Bacch. 333-334)

[Tão grandes riquezas tem; não sabe o que fazer com o ouro] 
(b) credo hercle adueniens nomen mutabit mihi

facietque extemplo Crucisalum me ex Chrysalo

(Plaut. Bacch. 361-362)

[Creio que retornando ele mudará meu nome e imediatamente me transformará de Crísalo em Pula-cruz]

Em (a), tanto habet quanto nescit estão no indicativo e não estão conectados por nenhum tipo de conjunção, de modo que não se pode falar em subordinação, no âmbito sintático-formal. Todavia, percebemos uma relação de causa e consequência entre esses verbos, o que é enfatizado pelo intensificador tantas, comum quando há uma oração subordinada consecutiva. Em (b), o verbo credo está justaposto aos verbos no indicativo mutabit e faciet, de modo que os elementos que comumente caracterizam a subordinação não estão presentes. Inclusive podemos traduzi-lo tanto como o fizemos, usando a subordinação no português, ou como se o credo fosse o que se chama de parentética, um trecho que comenta outro. $\mathrm{O}$ fato é que, mesmo havendo interdependência semântica, há a ausência de marcas morfossintáticas que as explicitem e enfatizem (como a conjunção ou o subjuntivo). Esse tipo de relação não me interessará. ${ }^{3}$

(2) Hipotaxe (marcada com conjunção):

Fac iam Amphitruonem aduenientem ab aedibus ut abigas (Plaut. Amph. 978)

[Trata de afastar Anfitrião da casa, quando ele chegar]

(3) "Parataxe" ou justaposição:

I sane, et quantum potest parata fac sint omnia (Plaut. Amph. 971)

[Vai sim e faz com que tudo esteja preparado o quanto antes possível]

${ }_{3}$ Cf. Weissenhorn (1884), que se detém somente sobre esse assunto. 
Enquanto em (2) há a conjunção subordinativa ut e o uso do subjuntivo abigas, em (3) o subjuntivo passivo parata sint encontra-se justaposto ao verbo principal fac, ou seja, temos a presença de ut em (2) e sua ausência em (3).

Para generalizar, tanto a hipotaxe quanto a justaposição são modos de organização de estruturas que, num primeiro momento, são vistas como apresentando a relação de subordinação, em que há um verbo tradicionalmente chamado de principal e um verbo, obrigatoriamente no subjuntivo, chamado de subordinado, núcleo de uma oração que exerce alguma função sintática ligada ao verbo principal, ou seja, seria equivalente a um sujeito (nominativo) ou a um objeto (acusativo), por exemplo. A diferença entre hipotaxe e justaposição, repito, seria respectivamente a presença e a ausência da conjunção ut.

Quando essas orações equivalem a um nominativo ou a um acusativo, recebem o nome de Oração Completiva ou Oração Substantiva. 4 Desse modo, chamo de ut completivo a conjunção que introduz orações de subjuntivo que ocupam o lugar de nominativos ou acusativos de outros verbos e sua ausência chamo de justaposição, já que um subjuntivo estará justaposto a outro verbo.

\section{Discutindo a bibliografia}

A bibliografia que comenta a existência dessas estruturas justapostas com subjuntivo ou o uso da conjunção ut é ampla e remonta ao menos à segunda metade do século XIX, como o estudo de Lindsay, Syntax of Plautus (1907, p. 66). A seguir, comento o estudo de dois pesquisadores, Durham e Bennett, que foram selecionados para comentário por serem os únicos que dispõem de coleções de exemplares que visavam ser exaustivas, enquanto todos os outros estudos a que tive acesso são impressionísticos, baseados na experiência de leitura dos pesquisadores e apresentam poucos exemplos, o que não diminui o valor global das análises e sistematizações, mas deixa de aproveitar a força argumentativa que um olhar detalhado e completo sobre a materialidade do texto permite. Vejamos como métodos e objetivos desses dois autores, que fizeram recolhas exaustivas dos dados, divergem dos que objetivo.

\footnotetext{
4 Veja, por exemplo, Cart et al. (2007, p. 130-135), Allen e Greenough (1903, § 560) e Faria (1958, p. 403-406).
} 
O estudo de Durham, The Subjunctive Substantive Clauses in Plautus, Not Including Iindirect Questions (1901), é o principal estudo para a questão deste trabalho, na medida em que seus objetos são justamente os mesmos, as orações substantivas subjuntivas. Todavia, tanto os objetivos quanto a metodologia de Durham são bem diferentes da aqui adotada. O autor teve a orientação de Bennett, o qual por sua vez utilizou a pesquisa de Durham em seu livro Syntax of Early Latin5 (1982), de modo que, ao compartilharem a mesma visão sobre o assunto, muito do que aqui for comentado também será encontrado no comentário sobre o livro de Bennett mais à frente. ${ }^{6}$

Sua preocupação fundamental é com a origem dessas construções, de modo que organiza as orações substantivas tendo como critério o entendimento de Delbrück sobre os valores semânticos do subjuntivo e do optativo indoeuropeus:

Chapter I. Substantive clauses developed from the jussive;

Chapter II. Substantive clauses developed from the subjunctive of determined resolution;

Chapter III. Substantive clauses developed from the deliberative subjunctive;

Chapter IV. Substantive clauses developed from the optative subjunctive;

Chapter V. Substantive clauses developed from the subjunctive of contingent futurity.7

Essa divisão de acordo com o valor original do subjuntivo fez com que um mesmo verbo principal aparecesse em mais de um capítulo.

Cada capítulo foi subdividido pelo autor especialmente de acordo com a semântica do verbo principal ou por expressão e organiza os exemplares de acordo com a presença ou ausência de um subordinador, com a ordem em que aparecem os verbos e com o fato de o exemplar apresentar um uso original (por exemplo, "clauses in which the subjunctive may be conceived as having been at

\footnotetext{
5 Publicado originalmente entre 1910 e 1914.

${ }^{6}$ No prefácio do livro de Durham (1901), lê-se: "The following study was undertaken at the suggestion of Professor Charles E. Bennett, and has throughout received his most generous attention".

7 Em tradução: "Capítulo I. Orações substantivas desenvolvidas do jussivo; Capítulo II. Orações substantivas desenvolvidas do subjuntivo de resolução determinada; Capítulo III. Orações substantivas desenvolvidas do subjuntivo deliberativo; Capítulo IV. Orações substantivas desenvolvidas do subjuntivo optativo; Capítulo V. Orações substantivas desenvolvidas do subjuntivo de futuro contingente".
} 
one time an independent jussive in paratatic use", ${ }^{8}$ Durham, 1901, p. 10) ou um uso derivado (por exemplo, "subjunctives where the assumption of an original independent jussive is impossible”, 9 Durham, 1901, p. 10). Em sua exposição, não são absolutamente claros os critérios para fazer essa distinção entre original e derivado. Independentemente disso, sua preocupação é completamente diversa da deste estudo, em que busco principalmente uma análise sincrônica do latim arcaico.

Além disso, o objetivo fundamental de meu estudo, a presença ou ausência do ut é declaradamente irrelevante para o estudo do autor:

But whatever meaning we may advocate as original for $u t$, its presence or absence as a special sign of subordination in a substantive clause is immaterial to the question of coordination or subordination between the parts. [...] The original difference between volo abeas and volo ut abeas must have been approximately that between 'I want you to go away' and 'I want you just to go away (Durham, 1901, p. 7).10

Ainda assim, sua listagem foi importantíssima na consolidação do corpus, cujos dados globais apresento mais à frente.

Como dito, o estudo de Durham é utilizado por Bennett, em Syntax of early Latin (1982). A partir da página 208 do volume 1 de sua obra, o autor se debruça sobre a parataxe no latim arcaico. No que diz respeito a Plauto, diz seguir

\footnotetext{
8 Em tradução: "orações nas quais o subjuntivo pode ser concebido como tendo sido em algum momento um jussivo independente em uso paratático". Sua definição de parataxe vem direto de Bennett (Critique of Some Recent Subjunctive Theories apud Durham, 1901, p. 4): "In order to exhibit parataxis, the two sentences assumed to have paratactic relation must each be capable of possessing an independent value. Just so soon as one of the two clauses is not capable of functioning alone, but only in conjunction with its neighbour, we have subordination or hypotaxis". Em tradução: "A fim de exibir parataxe, as duas sentenças que se supõem ter uma relação paratática devem, cada uma, ser capazes de possuir um valor independente. Tão logo uma das duas orações não é capaz de funcionar sozinha, mas somente em conjunção com sua vizinha, nós temos subordinação ou hipotaxe.”. Entretanto, não é claro no estudo de Durham quais critérios fazem ser possível discernir se duas sentenças podem existir independentemente ou não, o que torna a distinção bem subjetiva.

9 Em tradução: "subjuntivos dos quais é impossível se pressupor um jussivo original independente".

10 Em tradução: "Mas qualquer que seja o significado que podemos advogar como original para $u t$, sua presença ou ausência como um sinal especial de subordinação em uma oração substantiva é imaterial para a questão da coordenação ou subordinação entre as partes. [...] A diferença original entre volo abeas e volo ut abeas deve ter sido aproximadamente aquela entre 'Eu que que você vá' e 'Eu quero que você só vá'."
} 
Durham (1901) e adiciona a isso exemplos de Terêncio e outros escritores do latim arcaico. Vejamos seu entendimento do termo "parataxe":

In most primitive stages of language there are no formal subordinate clauses. Subordinate relations, however, exist, being indicated by the juxtaposition of independent sentences [...]. The name 'parataxis' is used to designate the primitive grammatical structure above referred to. [...]

In a narrower sense the name 'parataxis' is applied to those specimens of paratactic structure which ultimately develop in the history of a given language into subordinate clauses. Hypotaxis is merely another name for subordination, convenient as marking the contrast with its opposite parataxis.

It must not be thought, however, that the absence of a special subordinating particle is necessarily the mark of a paratactic form of expression and that where such particles are lacking we are to recognize independent uses. The subordinate relation often established itself without the help of special subordinate conjunctions (Bennett, 1987, p. 208-9). ${ }^{11}$

Em outras palavras, o termo parataxe aplicado ao latim arcaico é equivalente a justaposição, na medida em que não seria mais paratático de fato, como, na visão do autor, seria o caso da língua originalmente, mas relíquias que, sendo já subordinadas, não utilizam um conectivo: "In Early Latin we find many such subordinate clauses without an introductory conjunction. While these were undoubtedly at one time at the paratactic stage, they are at present best regarded as but relics of an earlier parataxis" (Bennett, 1987, p. 209). ${ }^{12}$

${ }^{11}$ Em tradução: "Nos estágios mais primitivos de uma língua não há formalmente orações subordinadas. Relações subordinadas, todavia, existem, sendo indicadas pela justaposição de sentenças independentes [...]. O nome 'parataxe' é usado para designar a estrutura gramatical primitiva acima referida. [...]

Num sentido mais estrito, o nome 'parataxe' é aplicado àqueles exemplares de estrutura paratática que ao fim se desenvolvem, na história de uma dada língua, em orações subordinadas. Hipotaxe é meramente outro nome para subordinação, conveniente para marcar o contraste com seu oposto, a parataxe.

Não se deve pensar, todavia, que a ausência de uma partícula subordinante especial é necessariamente a marca de uma forma paratática de expressão e que onde não se encontram tais partículas devemos reconhecer usos independentes. A relação subordinada frequentemente se estabeleceu sem a ajuda de conjunções subordinadas especiais".

12 Em tradução: "Em latim arcaico, nós encontramos muitas dessas orações subordinadas sem uma conjunção introdutória. Enquanto elas estiveram sem dúvida em algum momento no estágio paratático, são, no momento, melhor consideradas somente como relíquias de uma antiga parataxe.”. 
Nesse sentido, o estudo das orações com subjuntivo justapostas particularmente lhe interessa na medida em que ofereceriam a oportunidade de acessar características sintáticas de um latim muito antigo e inacessível.

Todavia, há uma importante questão metodológica, na medida em que Bennett tem alguns pressupostos não avalizados pela linguística contemporânea. O autor, explicando a sintaxe do latim arcaico, entende que as línguas passam por trajetórias evolutivas, de momentos mais primitivos a outros mais sofisticados. Veja à página 208: "In course of time every language develops certain types of independent sentences into subordinate clauses". ${ }^{13}$ Sendo assim, seu objetivo é fazer uma teoria da origem, ${ }^{14}$ buscando exemplos de períodos paratáticos que, segundo seu juízo, são primitivos e a partir dos quais, por analogia, outros exemplos são extensões: "Our object will be to determine as far as possible the original paratactic usage out of which the several varieties of subjunctive constructions have arisen, and to give the material belonging under each" (Bennett, 1987, p. 209). 15

Inclusive, algo que é fundamental para meu trabalho, as distinções entre períodos que usam o ut e períodos que o não usam, é desprezado por ele, por ser irrelevante a seu objetivo: "Whether $u t$ is present or absent is immaterial to our present purpose" (Bennett, 1987, p. 210), ou seja, "o ut estar presente ou ausente é imaterial para nosso presente propósito”. Ut seria apenas uma partícula adverbial enfática.

O elemento que o autor ignora é o quanto que a distribuição sincrônica da presença ou ausência da conjunção pode também ser indicativo de desenvolvimentos históricos. Mais à frente apresento apenas os dados globais, mas sua análise qualitativa, em subgrupos a partir de critérios específicos, pode tanto explicar o uso sincrônico como oferecer mais subsídios para análises históricas. Não tendo ainda os dados completamente depurados qualitativamente, não entrarei nessas análises, discutindo apenas uma visão geral quantitativa da distribuição de usos.

\footnotetext{
13 Em tradução: "No curso do tempo, toda língua desenvolve certos tipos de sentenças independentes em orações subordinadas.”.

14 No original, "theory of origin" (Bennett, 1987, p. 212).

${ }^{15}$ Em tradução: "Nosso objetivo será determinar, o tanto quanto possível, o uso paratático original a partir do qual as muitas variedades de construções subjuntivas surgiram e dar o material que pertence a cada uma [dessas variedades]".
} 
Assim, o trabalho de Bennett é importante para esta pesquisa por ser uma recolha dos exemplares com essas estruturas, abrangendo o latim arcaico, mas nada acrescenta no que diz respeito ao seu cerne, a existência ou não de regras que rejam a presença ou ausência do ut completivo no latim arcaico a partir da visualização da distribuição sincrônica do uso da estrutura.

Complementação com subjuntivo de facio e uolo

É muito comum na bibliografia, haver a organização das orações substantivas ou completivas, de acordo com a semântica do verbo principal (e.g. Dahl, 1882; Weissenhorn, 1884; Durham, 1901; entre outros).

Em sua gramática, Allen e Greenough (1903, § 562) também o fazem. Classificam as orações substantivas em: orações de indicativo com quod; interrogativas indiretas; orações de infinitivo; e orações de subjuntivo. Este último tipo, o qual me interessa, é subdividido por eles em: de finalidade (“of purpose”, o que complementa com "command, wish, fear") e de resultado ("of result", o que complementa com "happen, effect, etc.”; também chamado por eles no título do parágrafo 567 de orações consecutivas, “consecutive clauses”). A fim de oferecer conclusões acerca de ambos tipos semânticos de orações de subjuntivo sugeridos por essa tradição, esta pesquisa tem como foco verificar o uso desse tipo de oração com os verbos principais uolo e facio, respectivamente os principais representantes das categorias supramencionadas "de finalidade" e "de resultado", e que também são verbos de alta frequência, o que permite que se garantam dados suficientes para embasar qualquer conclusão.

\section{Resultados globais consolidados}

Como o objetivo da pesquisa é analisar a presença e ausência do ut de modo quantitativo, tenho como unidade de contagem os subjuntivos que acompanham os verbos facio e uolo das 21 peças de Plauto, principal corpus do latim arcaico que nos restou.

Os exemplares foram recolhidos inicialmente com a ferramenta de pesquisa Diogenes e, então, comparei minha listagem inicial com as listagens similares de Durham (1901) e de Lodge (1971). Minha principal referência ao texto de 
Plauto foram os livros da Loeb Classical Library atualizados em edição recente (Plautus, 2011a, 2011b, 2011c, 2012, 2013). Além disso, consultei comentários de cada uma das peças, importantes em auxiliar na interpretação das estruturas, e edições críticas para avaliar as soluções textuais variadas. Após esses processos, pude fixar o corpus, cujos números apresento a seguir.

A Tabela 1 resume a quantidade de todos os exemplares de complementação com subjuntivo do verbo facio, organizados segundo a ausência ou presença da conjunção $u t$, em cada peça de Plauto. São apresentadas também as frequências relativas por peça. Ao final, temos os resultados totais.

Os resultados totais em Plauto foram os seguintes: 35,92\% dos exemplares (88 de 245) apresentam justaposição, enquanto, complementarmente, 64,08\% (157 de 245) apresentam a conjunção $u t$.

Tabela 1: Frequência de todas as ocorrências de complementação subjuntiva do verbo facio em cada peça de Plauto de acordo com a ausência ou presença da conjunção ut

\begin{tabular}{|c|c|c|c|c|c|}
\hline & $\begin{array}{l}\text { Justa- } \\
\text { posto }\end{array}$ & Com ut & Total & $\mathrm{J} / \mathrm{T}$ & $\mathrm{ut} / \mathrm{T}$ \\
\hline Amphitruo & 10 & 22 & 32 & $31,25 \%$ & $68,75 \%$ \\
\hline Asinaria & 3 & 7 & 10 & $30,00 \%$ & $70,00 \%$ \\
\hline Aulularia & 3 & 9 & 12 & $25,00 \%$ & $75,00 \%$ \\
\hline Bacchides & 4 & 5 & 9 & $44,44 \%$ & $55,56 \%$ \\
\hline Captiui & 2 & 12 & 14 & $14,29 \%$ & $85,71 \%$ \\
\hline Casina & 5 & 4 & 9 & $55,56 \%$ & $44,44 \%$ \\
\hline Cistellaria & o & 4 & 4 & $0,00 \%$ & $100,00 \%$ \\
\hline Curculio & 4 & 6 & 10 & $40,00 \%$ & $60,00 \%$ \\
\hline Epidicus & 2 & 1 & 3 & $66,67 \%$ & $33,33 \%$ \\
\hline Menaechmi & 8 & 4 & 12 & $66,67 \%$ & $33,33 \%$ \\
\hline Mercator & 3 & 6 & 9 & $33,33 \%$ & $66,67 \%$ \\
\hline Miles Gloriosus & 4 & 11 & 15 & $26,67 \%$ & $73,33 \%$ \\
\hline Mostellaria & 5 & 6 & 11 & $45,45 \%$ & $54,55 \%$ \\
\hline Persa & 5 & 12 & 17 & $29,41 \%$ & $70,59 \%$ \\
\hline Poenulus & 7 & 13 & 20 & $35,00 \%$ & $65,00 \%$ \\
\hline Pseudolus & 8 & 12 & 20 & $40,00 \%$ & $60,00 \%$ \\
\hline Rudens & 4 & 9 & 13 & $30,77 \%$ & $69,23 \%$ \\
\hline Stichus & 2 & 6 & 8 & $25,00 \%$ & $75,00 \%$ \\
\hline Trinummus & 5 & 5 & 10 & $50,00 \%$ & $50,00 \%$ \\
\hline Truculentus & 4 & 3 & 7 & $57,14 \%$ & $42,86 \%$ \\
\hline Vidularia & O & $\mathrm{O}$ & $\mathrm{O}$ & $0,00 \%$ & $0,00 \%$ \\
\hline Total: & 88 & 157 & 245 & $35,92 \%$ & $64,08 \%$ \\
\hline
\end{tabular}


Dada a amostra de 245 exemplares, há 95\% de probabilidade de a proporção da população (ou seja, do latim de Plauto como um todo) que apresenta ut estar no seguinte intervalo de confiança: entre 58,28\% e 70,29\%. ${ }^{16}$ Isso reforça a hipótese de Plauto como um todo e não somente nessas 21 peças preferir a complementação subjuntiva com ut à justaposição.

Olhando detidamente para os dados de cada uma das peças, vejamos que Vidularia não apresentou nenhum exemplar, algo aceitável visto o estado incompleto do texto, e Cistellaria apresentou 100\% de uso com ut, mas com apenas 4 exemplares, o que torna seu resultado pouco relevante. Das 19 peças restantes, 15 apresentam a preferência pelo ut completivo como tendência principal, variando de $54,55 \%$ de Mostellaria a $85,71 \%$ no Captiui. De modo complementar, nesse grupo o uso de justaposição varia entre 14,29\% em Captiui a 45,45\% em Mostellaria.

A exceção de Casina, Epidicus, Menaechmi, Trinummus, Truculentus pode ser facilmente explicada pelo baixo volume de dados na visão individual de cada peça, o que faz a amostra ser menos representativa, em oposição ao conjunto de dados de todas as peças.

Gostaria ainda de ressaltar que as peças com maior número de exemplares (Amphitruo [32], Pseudolus [20] e Poenulus [20]) apresentam uma proporção próxima da do total, respectivamente $68,75 \%, 60,00 \%$ e 65,00\%, como é de se esperar que aconteça. ${ }^{17}$

A primeira conclusão que esses dados oferecem é que a língua de Plauto já demonstra um estado avançado da generalização da conjunção ut, no que se refere ao verbo facio, já que o estado inicial pré-literário seria somente a justaposição, enquanto que no latim clássico haveria a generalização do uso.

A Tabela 2 resume a quantidade de todos os exemplares de complementação com subjuntivo do verbo uolo, organizados segundo a ausência ou presença da conjunção ut, em cada peça de Plauto. São apresentadas também as frequências relativas por peça. Ao final, temos os resultados totais.

\footnotetext{
${ }^{16}$ A partir da fórmula para o intervalo de confiança, oferecida por Woods, Fletcher e Hughs (1996, p. 99-101).

${ }_{17}$ Cf. os capítulos iniciais de Woods, Fletcher e Hughs (1996).
} 
Os resultados totais em Plauto foram os seguintes: 67,50\% dos exemplares (81 de 120) apresentam justaposição, enquanto, complementarmente, 32,50\% (39 de 120) apresenta a conjunção $u t$.

Dada a amostra de 120 exemplares, há 95\% de probabilidade de a proporção da população (ou seja, do latim de Plauto como um todo) que apresenta ut estar no seguinte intervalo de confiança: entre 24,54\% e 41,30\%. ${ }^{18}$ Isso reforça a hipótese de Plauto como um todo e não somente nessas 21 peças preferir a complementação subjuntiva justaposta à com $u t$.

Tabela 2: Frequência de todas as ocorrências de complementação subjuntiva do verbo uolo em cada peça de Plauto de acordo com a ausência ou presença da conjunção ut

\begin{tabular}{|c|c|c|c|c|c|}
\hline & $\begin{array}{l}\text { Justa- } \\
\text { posto } \\
\end{array}$ & Com ut & Total & $\mathrm{J} / \mathrm{T}$ & $\mathrm{ut} / \mathrm{T}$ \\
\hline Amphitruo & 1 & 3 & 4 & $25,00 \%$ & $75,00 \%$ \\
\hline Asinaria & 2 & 1 & 3 & $66,67 \%$ & $33,33 \%$ \\
\hline Aulularia & 2 & 2 & 4 & $50,00 \%$ & $50,00 \%$ \\
\hline Bacchides & 4 & 2 & 6 & $66,67 \%$ & $33,33 \%$ \\
\hline Captiui & 7 & 1 & 8 & $87,50 \%$ & $12,50 \%$ \\
\hline Casina & 4 & 1 & 5 & $80,00 \%$ & $20,00 \%$ \\
\hline Cistellaria & 1 & 1 & 2 & $50,00 \%$ & $50,00 \%$ \\
\hline Curculio & 1 & O & 1 & $100,00 \%$ & $0,00 \%$ \\
\hline Epidicus & 2 & 4 & 6 & $33,33 \%$ & $66,67 \%$ \\
\hline Menaechmi & 2 & 1 & 3 & $66,67 \%$ & $33,33 \%$ \\
\hline Mercator & 5 & 2 & 7 & $71,43 \%$ & $28,57 \%$ \\
\hline Miles Gloriosus & 4 & 4 & 8 & $50,00 \%$ & $50,00 \%$ \\
\hline Mostellaria & 3 & 2 & 5 & $60,00 \%$ & $40,00 \%$ \\
\hline Persa & 3 & 3 & 6 & $50,00 \%$ & $50,00 \%$ \\
\hline Poenulus & 14 & 1 & 15 & $93,33 \%$ & $6,67 \%$ \\
\hline Pseudolus & 6 & 6 & 12 & $50,00 \%$ & $50,00 \%$ \\
\hline Rudens & 5 & 3 & 8 & $62,50 \%$ & $37,50 \%$ \\
\hline Stichus & 6 & O & 6 & $100,00 \%$ & $0,00 \%$ \\
\hline Trinummus & 4 & O & 4 & $100,00 \%$ & $0,00 \%$ \\
\hline Truculentus & 5 & 2 & 7 & $71,43 \%$ & $28,57 \%$ \\
\hline Vidularia & O & $\mathrm{O}$ & $\mathrm{O}$ & $0,00 \%$ & $0,00 \%$ \\
\hline Total: & 81 & 39 & 120 & $67,50 \%$ & $32,50 \%$ \\
\hline
\end{tabular}

\footnotetext{
${ }^{18}$ A partir da fórmula para o intervalo de confiança, oferecida por Woods, Fletcher, Hughs (1996, p. 99-101).
} 
Olhando detidamente para os dados de cada uma das peças, vejamos que Vidularia não apresentou nenhum exemplar, algo aceitável visto o estado incompleto do texto. Por sua vez, uma análise dos números peça a peça não é muito interessante, visto que muitas delas apresentam pouquíssimos exemplares; somente a soma total traz um número significativo de exemplares. Ainda assim, é notável que das 20 peças que apresentam algum dado, somente Amphitruo (25\% de justaposição) e Epidicus (33,33\% de justaposição) apresentam menos exemplares justapostos do que com $u t$.

Ao contrário do que vimos com o verbo facio, o verbo uolo prefere a justaposição em 7 de 10 exemplares, o que a princípio permitiria a conclusão de que o processo de generalização da conjunção ut não estava ocorrendo com o verbo uolo. Todavia, somente uma análise qualitativa, ainda a ser feita, permitirá que verifiquemos os contextos em que o ut é utilizado e se há alguma explicação para esse quadro.

\section{Conclusão}

Enquanto boa parte da bibliografia, visando cobrir uma ampla gama de estruturas do latim arcaico, se contenta com poucos exemplares ilustrativos dessas estruturas, as duas pesquisas que apresentam de certo modo esses dados têm outros objetivos e outros pressupostos teóricos. Em todos os casos, as análises sobre as estruturas costumam ignorar a presença ou ausência da conjunção ut nas orações completivas e trazer análises que abarquem todos os verbos principais juntos. Os dados que apresentei acima indicam que o comportamento sincrônico da distribuição do uso ou não da conjunção pode variar muito, sendo inclusive opostos como no caso dos verbos facio e uolo. Vejamos um resumo na Tabela 3:

Tabela 3: Frequência da presença e ausência do ut completivo nos verbos facio e uolo em Plauto

\begin{tabular}{llll}
\hline & Justaposto & Com ut & Total \\
\hline facio & $35,92 \%(88)$ & $64,08 \%(157)$ & $100 \%(245)$ \\
uolo & $67,50 \%(81)$ & $32,50 \%(39)$ & $100 \%(120)$ \\
\hline
\end{tabular}


Apenas o tabelamento exaustivo dos dados permitiu essa visão de modo preciso e a quantidade estatisticamente relevante de dados permite a hipótese de que esse resultado não se restringe às 21 peças, mas à língua de Plauto como um todo.

Assim, a situação de uso ou não da conjunção ut para introduzir um complemento com verbo no subjuntivo depende muito de qual é o verbo ou a estrutura principal. E mesmo considerando o mesmo verbo principal, análises preliminares indicam que há regras subjacentes ao uso ou não da conjunção. Todavia, qualquer afirmação nesse sentido exige que os dados sejam trabalhados em uma análise qualitativa que os organizem em subgrupos, a fim de se verificar quais seriam essas regras situacionais que regem essa escolha, e quais contextos são irrelevantes para isso.

Artigo recebido em 31.12.2017, aprovado em 01.02.2018. 


\section{REFERÊNCIAS BIBLIOGRÁFICAS}

Fontes Primárias

Plautus. Amphitryon. The Comedy of Asses. The Pot of Gold. The Two Bacchises. The Captives. Editado e traduzido por Wolfgang de Melo. Cambridge MA, London: Harvard University Press, 2011a. v. I. (Loeb Classical Library 60).

. Casina. The Casket Comedy. Curculio. Epidicus. The Two Menaechmuses. Editado e traduzido por Wolfgang de Melo. Cambridge MA, London: Harvard University Press, 2011b. v. II. (Loeb Classical Library 61).

. The Merchant. The Braggart Soldier. The Ghost. The Persian. Editado e traduzido por Wolfgang de Melo. Cambridge MA, London: Harvard University Press, 2011c. v. III. (Loeb Classical Library 163).

. The Little Carthaginian. Pseudolus. The Rope. Editado e traduzido por Wolfgang de Melo. Cambridge MA, London: Harvard University Press, 2012. v. IV. (Loeb Classical Library 260).

. Stichus. Three-Dollar Day. Truculentus. The Tale of a Traveling-Bag. Fragments. Editado e traduzido por Wolfgang de Melo. Cambridge MA, London: Harvard University Press, 2013. v. V. (Loeb Classical Library 328).

\section{Bibliografia crítica}

Allen, J. H.; Greenough, J. B. New Latin Grammar. Boston, London: Ginn \& Company, 1903.

Bennett, C. E. Syntax of early Latin. Hildesheim: Georg Olms, 1982 [1910]. 2 v. em 1.

Cart, A. et al. Grammaire Latine. [S.l.]: Nathan, 2007 [1955].

Dahl, B. Die lateinische Partikel ut. Kristiania [Oslo]: Druck von Grøndahl \& Søn, 1882.

Diogenes. Disponível em: <https://community.dur.ac.uk/p.j.heslin/Software/Diogenes/>. Acesso em: o5 dez. 2017.

Durham, C. L. The subjunctive substantive clauses in Plautus, not including indirect questions. Ithaca (N.Y.): Cornell University Press, 1901. (Cornell Studies In Classical Philology, v. 13). Disponível em: <http://hdl.handle.net/2027/mdp.39015035972697>. Acesso em: o5 dez. 2017.

Faria, E. Gramática superior da língua latina. Rio de Janeiro: Livraria Acadêmica, 1958.

Lindsay, W. M. Syntax of Plautus. Oxford: James Parker and Co., 1907.

. T. Macci Plavti comoediae: recognovit breviqve adnotatione critica instruxit. Grã-bretanha: Oxonii e Typographeo Clarendoniano, 1903. v.1 (Amphitruo - Mercator).

. T. Macci Plavti comoediae: recognovit brevique adnotatione critica instruxit. Grã-bretanha: Oxonii e Typographeo Clarendoniano, 1905, v. 2 (Miles Gloriosus - Fragmenta). 
Lodge, G. Lexicon Plautinum. Hildesheim; New York: Georg Olms Verlag, 1971, $2 \mathrm{v}$.

Weiss, M. Outline of the Historical and Comparative Grammar of Latin. Ann Arbor; New York: Beech Stave Press, 2009.

Weissenhorn, J.B. Parataxis Plautina. Burghausen: M. Speth, 1884.

Woods, A.; Fletcher, P.; Hughs, A. Statistics in Language Studies. Cambridge: Cambridge University Press, 1996 [1986]. 
